\title{
Out-of-field Generic ML Training with In-field Specific Adaptation to Facilitate ML Deployments
}

\author{
Behnam Shariati, Marc Ruiz, and Luis Velasco* \\ Optical Communications Group (GCO), Universitat Politècnica de Catalunya (UPC), Barcelona, Spain \\ e-mail: lvelasco@ac.upc.edu
}

\begin{abstract}
A two-phase strategy to facilitate ML algorithm deployment in real networks is demonstrated: out-of-field training uses data from simulation and testbed experiments with generic equipment whereas in-field adaptation is applied to support heterogeneous equipment. (C) 2019 Optical Society of America
\end{abstract}

OCIS codes: (060.0060) Fiber optics and optical communications, (060.1155) All optical networks

\section{Introduction}

The use of cost-effective Optical Spectrum Analyzers (OSA) as a tool for the detection and identification of soft-failures degrading the Quality of Transmission of optical signals has been recently proposed in [1]. Machine Learning (ML) applied to the spectra acquired by OSAs installed in intermediate nodes has been successfully demonstrated for laser drift and/or filter-related failures, e.g., filter shift (FS), and tight filtering (FT), which deform the shape of the optical spectrum in a noticeable way. As filter cascading effects produce effects similar to FT, ML approaches were improved in [2] where the residual-based approach was proposed; it is based on analyzing the residual signal obtained by subtracting the signal acquired by OSAs from an expected signal synthetically generated at every intermediate node.

Notwithstanding the noticeable results, one single filter type was considered in [1], [2], which limits the deployment of ML approaches to real operator networks that usually consist of equipment from different vendors. The most straightforward solution to overcome this limitation is to have different models being trained upon various types of filters that might be available in the network. Nonetheless, it makes the training phase very complex and data-hungry. Yet, it will not be easy to comprehend the sequence of filters a priori and the responses of a slightly non-identical filter in the network might not be very well detected, necessitating even more combination of models to have an appropriate generic model.

In this paper, we propose a two-phase strategy to facilitate ML algorithm deployment in real networks; it consists in: i) training accurate models for a reference filter type based on simulations and/or experiments carried out in laboratory or test-bed facilities and ii) devising a proper adaptation mechanism that makes adjustments on the data for the specific signal being analyzed, which might have traversed different filter types along its route from the transmitter. Note that this strategy also facilitates the introduction of new filter types, as current vendors deploy new equipment releases in the network. The residual-based approach, due to its dependency on the synthetic behavior of the filter responses, has potential characteristics to get adapted to different types of filter. We present an enhanced version of such approach with the particular capability of getting adapted to new filter types in the network. This allows out-of-field model training and in-field adaptation, which makes it a robust feasible solution for networks with heterogeneous filtering.

\section{Residual-based training and adaptation for filter soft-failure detection and identification}

Fig. 1 overviews the proposed out-of-field ML training and in-field model adaptation strategy. Scenarios with one single type of filters (labeled F1 in Fig. 1a) are considered in simulation and/or lab experiments to produce a large dataset that is used for ML training purposes. When the ML model is deployed in the field, an adaptation procedure takes into account the specific types of filter that a given signal has passed through (Fig. 1b).

The residual-based approach lies in pre-processing the acquired optical spectrum by comparing it to the one that would be expected after passing the same number of filters than the signal. This comparison produces a

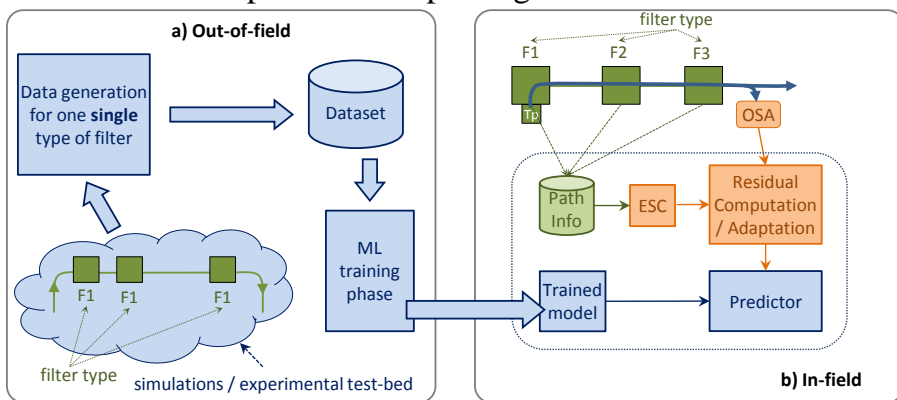

Fig. 1. Out-of-field ML training and in-field model adaptation
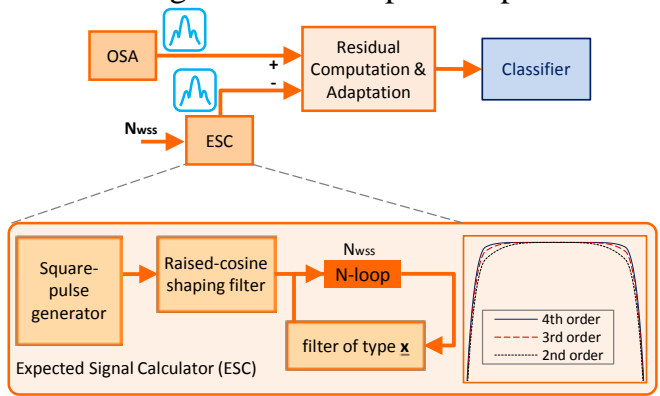

Fig. 2. Soft-failure detection based on residuals analysis

The research leading to these results has received funding from the EC through the METRO-HAUL project (G.A. $\mathrm{n}^{\circ}$ 761727), from the Spanish MINECO TWINS project (AEI/FEDER TEC2017-90097-R), and from the Catalan Institution for Research and Advanced Studies (ICREA). 
residual signal representing the differential deformation that it is used as an input for a classifier that detects soft-failures (Fig. 2). Two modules are required to compute the residual signal: $i$ ) the Expected Signal Calculator (ESC) and ii) the Residual Computation and Adaptation module [2]. The ESC module generates a theoretically-calculated optical spectrum emulating a properly operating lightpath. The aim of ESC module is to synthetically reproduce an averaged noise-free version of the measured optical signal. Then, the residual signal is easily obtained by subtracting the acquired signal by the OSA from the signal generated by the ESC module. However, further elaboration on the residual signal is required to make it suitable for decision-making and training the classifiers. Ultimately, the elaborated residual signals can be used to train Support Vector Machine based classifiers [3] to detect and identify filter failure cases.

The in-field adaptation is performed in $i$ ) the ESC module by considering the specific filters that the signal has passed through; see three filter transfer functions in Fig. 2, and ii) the residual computation module that normalizes and adapts the residuals for the signal under analysis. Next section describes residuals adaptation.

\section{Residual adaptation}

Following the procedure presented in [2], the calculated residual is normalized with respect to the mean value of the central part of the residual, so the mean becomes 0 . This normalization approach is operational when the same type of filter exists in both out-of-field training and in-the-field operation of the ML algorithm. However, when applied to other filter types it does not work well (see the results below). In view of this, we propose an adaptation procedure (Fig. 3a) that consists in dividing the residual signal in three segments (Fig. 3b) and apply different normalization methods to every segment, reflecting the filter characteristics; the normalization reference of every segment is obtained by applying linear regression to the un-normalized version of the residual signal obtained for that segment. Then, the residual computation and adaptation module receives the signal, as well as the linear regression coefficients modeling three different normalization references that consider the filter characteristics. For this stage, the number of adaptation mechanism loop equals to the number of filter types that the lightpath has passed through. By subtracting every segment of the un-normalized residuals from the corresponding normalization reference, a filter type-agnostic residual signal is obtained. Note that, as the amount of filter cascading effect depends on the transfer function of the filter, there might be an undesirable deviation in the residual signals when the lightpath traverses different filter types; this deviation is compensated in the fine tuning step. The amount of deviation can be computed locally assuming that the mean value of the residual remains zero when the signal is in proper operation mode. Ultimately, a single classifier trained with the measurements collected in the lab based on a reference filter type, can be used for optical spectra experiencing filtering effects from different types of filter.

The efficiency of this adaptation method is illustrated in Fig. 4. The residual signals of a lightpath passing through three different types of filters with Gaussian transfer function of order 2, 3, and 4 are illustrated in Fig. 4a. Normalization shifts the residuals so its mean to be 0 (Fig. 4b). Note that the differences among residuals are clearly seen at the edges, whereas they are virtually identical in the central part before and after normalization. Adaptation focus on compensating the effects of the different filters and the results are clearly visible at the edges (Fig. 4c); note that the most relevant parts of the residuals to detect filter-related soft-failures are that of the edges. As shown, even though the signals pass through different types of filters, they result in an identical residual signal, removing the filter-dependent characteristics of the residual signal.
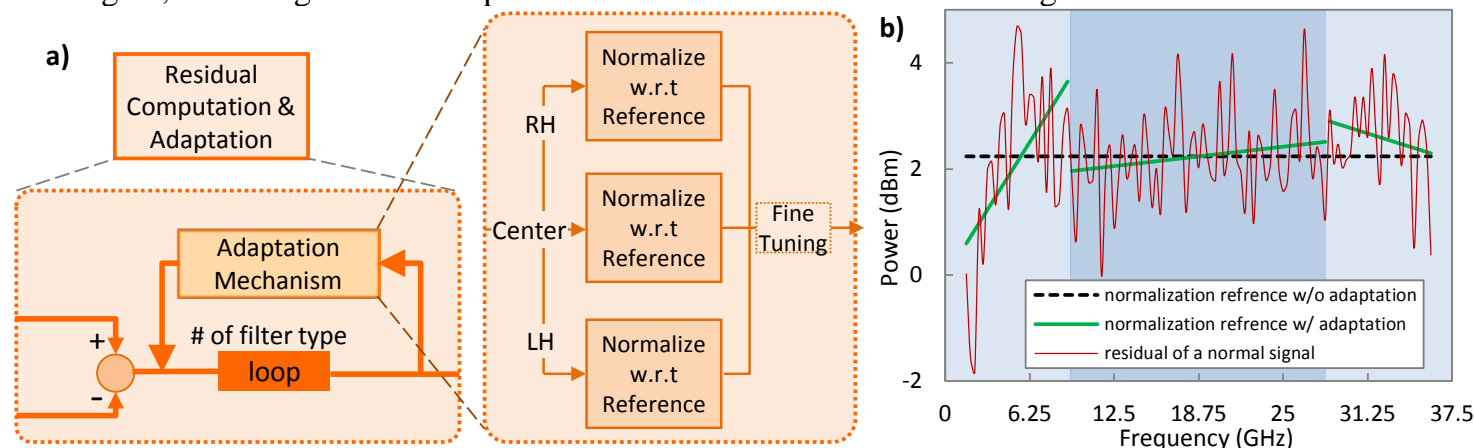

Fig. 3. a) Residual computation and adaptation mechanism and b) normalization references for $4^{\text {th }}$ order Gaussian.

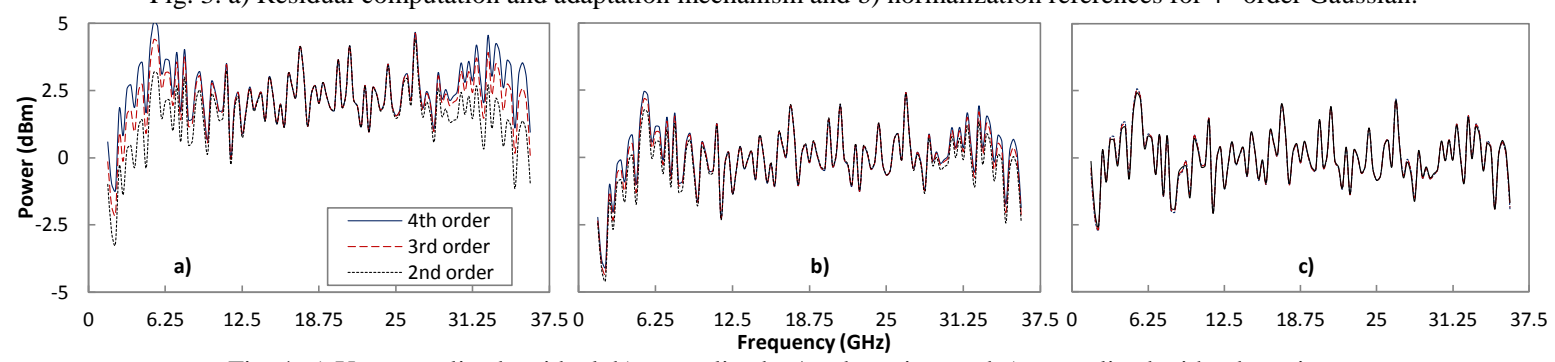

Fig. 4. a) Un-normalized residual, b) normalized w/o adaptation, and c) normalized with adaptation. 

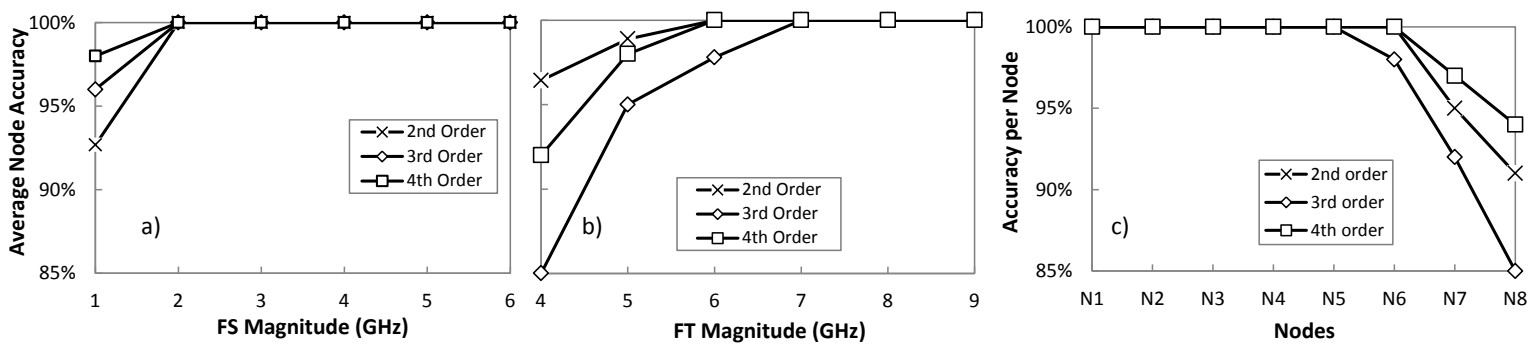

Fig. 5. Average node accuracy w.r.t failure magnitudes for a) FS and b) FT. c) accuracy per node w.r.t the sequence of cascaded nodes.

\section{Results}

In this section, we discuss the obtained results and demonstrate how the proposed adaptation mechanism enables the residual-based approach to be applied to optical spectrum of a signal after passing through different types of filters in the network. For the experiments, we configured a VPIPhotonics scenario where a $100 \mathrm{~Gb} / \mathrm{s}$ DP-QPSK modulated signal was emulated. After the transmitter, the optical signal passes through eight optical nodes (from N1 to N8); after every span, an optical amplifier compensates for the accumulated attenuation of the fiber. Every optical node consists of two Wavelength Selective Switches (WSS), each one modeled as a single optical filter with a $2^{\text {nd }}$ order Gaussian transfer function for the training phase; filters bandwidth is set to $37.5 \mathrm{GHz}$, leaving $7.5 \mathrm{GHz}$ as a guard band for the lightpath. Finally, the optical signal ends in a coherent receiver that compensates for the impairments introduced throughout the transmission. In addition, OSAs with 312.5 MHz resolution are placed after every optical node to acquire the optical spectrum of every optical link.

Aiming at emulating failure scenarios, we modified the characteristics of the $2^{\text {nd }}$ WSS of every node in the setup; its bandwidth and central frequency were modified to model FT and FS failures, respectively. A large dataset of failures was collected by inducing failures of magnitude in the range [1-8] GHz for FS and in the range [1-15] GHz for FT. We configured optical filters to be $2^{\text {nd }}$ order Gaussian for training and re-configured them to become $3^{\text {rd }}$ and $4^{\text {th }}$ order Gaussian for testing, where the same failure scenarios were simulated.

We looked firstly at the benefits of applying the adaptation mechanism for identifying the normal cases. We found that accuracy (number of correctly detected cases over the total number of cases) is very poor $(<20 \%)$ when no adaptation is applied and becomes perfect with residual adaptation. Next, we looked at the benefits of applying residual adaptation for detecting failures. Three cases were studied: i) $2^{\text {nd }}$ order for both out-of-field training and in-field testing; note that no adaptation is needed (the case in [2]), ii) $3^{\text {rd }}$ order and ii) $4^{\text {th }}$ order, in which $2^{\text {nd }}$ order filters were used for training and $3^{\text {rd }}$ and $4^{\text {th }}$ order, respectively $\mathrm{w} /$ adaptation were used for testing. The results are reported in Fig. 5, where Fig. 5(a-b) show the average node accuracy of identifying FS and FT, respectively, for failures in all 8 nodes and varying levels of failure magnitudes. The accuracy is promising for all the cases under study, even though it degrades for very small magnitudes in which the spectrum looks like normal cases; in fact, failure detection is $100 \%$ in all cases being the failure identification step the cause of the reduced accuracy (Table I). To highlight the impact of cascaded nodes, Fig. 5c presents the average accuracy for FS and FT with respect to the node where the failure occurs; failure magnitudes of range [1-4] GHz for FS and [4-7] GHz for FT were considered. As shown, the accuracy drops at the very last nodes as a result of accumulated filter cascading effects makes very challenging to distinguish between different cases.

Ultimately, the efficiency of the algorithm for transmission system with two different filter types was evaluated. To this end, we modified the abovedescribed setup to have $2^{\text {nd }}$ order Gaussian filters in the first 4 nodes and $4^{\text {th }}$ order Gaussian filters in the last 4 ones. As reported in Table I, failure detection accuracy is $100 \%$ while performance degradation happens when failure identification is executed. As a result, the minimum failure magnitude to be detected with $100 \%$ accuracy is 5 and $7 \mathrm{GHz}$, for FS and FT, respectively, just a bit higher than in the case of one single filter type, which validates the performance of the proposed residual adaptation method.

Table I. Result Comparisons

\begin{tabular}{|c|c|c|c|}
\hline \multirow{2}{*}{ Scenario } & Failure & \multicolumn{2}{|c|}{ Failure Type Identification } \\
\cline { 3 - 4 } & Detection & Min FS Magnitude & Min FT Magnitude \\
\hline only $2^{\text {nd }}$ or $4^{\text {th }}$ order & $100 \%$ & $2 \mathrm{GHz}$ & $6 \mathrm{GHz}$ \\
\hline only $3^{\text {rd }}$ order & $100 \%$ & $2 \mathrm{GHz}$ & $7 \mathrm{GHz}$ \\
\hline mix of $2^{\text {nd }}$ and $4^{\text {th }}$ order & $100 \%$ & $5 \mathrm{GHz}$ & $7 \mathrm{GHz}$ \\
\hline
\end{tabular}

\section{Conclusions}

ML algorithm can be trained out-of-field with measurements from testbeds and/or simulations using one single reference filtering solution (possibly belonging to a single vendor). A reliable in-field adaptation mechanism is demonstrated to enable heterogeneous filtering solutions (belonging to different vendors).

\section{References}

[1] A. P. Vela et al., "Soft Failure Localization during Commissioning Testing and Lightpath Operation,” IEEE/OSA JOCN, 2018

[2] B. Shariati et al., "Learning from the Optical Spectrum: Failure Detection and Identification [Invited],” IEEE/OSA JLT, 2018.

[3] D. Rafique and L. Velasco, "Machine Learning for Optical Network Automation: Overview, Architecture and Applications," IEEE/OSA JOCN, 2018. 